Article

\title{
Simulating Forest Fire Spread with Cellular Automation Driven by a LSTM Based Speed Model
}

\author{
Xingdong Li ${ }^{1,2, *} \mathbb{C}$, Mingxian Zhang ${ }^{1}{ }^{(D}$, Shiyu Zhang ${ }^{1}$, Jiuqing Liu ${ }^{1}$, Shufa Sun ${ }^{2,3}$, Tongxin $\mathrm{Hu}^{2,4}$ \\ and Long Sun 2,4 \\ 1 College of Mechanical and Electrical Engineering, Northeast Forestry University, Harbin 150040, China; \\ dljdzmx@nefu.edu.cn (M.Z.); 2018010083zsy@nefu.edu.cn (S.Z.); nefujdljq@163.com (J.L.) \\ 2 Northern Forest Fire Management Key Laboratory of the State Forestry and Grassland Bureau, \\ Northeast Forest University, Harbin 150040, China; ssfangel@163.com (S.S.); htxhtx@nefu.edu.cn (T.H.); \\ sunlong365@126.com (L.S.) \\ 3 College of Engineering and Technology, Northeast Forestry University, Harbin 150040, China \\ 4 College of Forestry, Northeast Forestry University, Harbin 150040, China \\ * Correspondence: lixd@nefu.edu.cn
}

Citation: Li, X.; Zhang, M.; Zhang, S.; Liu, J.; Sun, S.; Hu, T.; Sun, L. Simulating Forest Fire Spread with Cellular Automation Driven by a LSTM Based Speed Model. Fire 2022, 5, 13. https://doi.org/10.3390/fire 5010013

Academic Editors: Andrew T. Hudak and Luis A. Ruiz

Received: 8 December 2021

Accepted: 17 January 2022

Published: 20 January 2022

Publisher's Note: MDPI stays neutral with regard to jurisdictional claims in published maps and institutional affiliations.

Copyright: (C) 2022 by the authors. Licensee MDPI, Basel, Switzerland. This article is an open access article distributed under the terms and conditions of the Creative Commons Attribution (CC BY) license (https:// creativecommons.org/licenses/by/ $4.0 /$ )

\begin{abstract}
The simulation of forest fire spread is a key problem for the management of fire, and Cellular Automata (CA) has been used to simulate the complex mechanism of the fire spread for a long time. The simulation of CA is driven by the rate of fire spread (ROS), which is hard to estimate, because some input parameters of the current ROS model cannot be provided with a high precision, so the CA approach has not been well applied yet in the forest fire management system to date. The forest fire spread simulation model LSTM-CA using CA with LSTM is proposed in this paper. Based on the interaction between wind and fire, S-LSTM is proposed, which takes full advantage of the time dependency of the ROS. The ROS estimated by the S-LSTM is satisfactory, even though the input parameters are not perfect. Fifteen kinds of ROS models with the same structure are trained for different cases of slope direction and wind direction, and the model with the closest case is selected to drive the transmission between the adjacent cells. In order to simulate the actual spread of forest fire, the LSTM-based models are trained based on the data captured, and three correction rules are added to the CA model. Finally, the prediction accuracy of forest fire spread is verified though the KAPPA coefficient, Hausdorff distance, and horizontal comparison experiments based on remote sensing images of wildfires. The LSTM-CA model has good practicality in simulating the spread of forest fires.
\end{abstract}

Keywords: forest fire; LSTM; cellular automata; fire spread simulation; extreme learning machine

\section{Introduction}

Forest fire is a global natural disaster [1]. In recent years, the frequent occurrence of forest fires has been caused by global warming, the annual increase in the amount of combustible materials, and the difficulty controlling fire source [2,3]. There are many complex factors influencing the spread of forest fires [4,5]. Accurate simulation [6-10] of forest fire spread can effectively reduce casualties and property losses [11]. The rate of fire spread (ROS) model is one of the important measures for conducting the simulation of forest fire spread, including physical and quasi-physical models and empirical and quasi-empirical models. The Rothermel [12] and Wang Zhengfei [13] models are the most common methods for simulating the spread of forest fire. These two methods belong to the ROS model, whose parameters are optimized experimentally. Their practicality is generally not ideal. Taking the equation of Rothermel model as an example, its structure is complicated, and too many inputs are needed, so its application is restricted. The Wang Zhengfei model can be applicable to slope s below $60^{\circ}$. 
The Rothermel and Wang Zhengfei models can estimate the ROS at any point but do not have the direction of fire spread and cannot predict the shape and location of fire spread. Propagation methods must be used to represent the changes in fire contours, which are based on the method of vector and grid data. The Huygens principle is applied to the vector method, and the Cellular Automata (CA) is used in the grid data method. Farsite [14] is a classical simulation system that combines the Rothermel model and Huygens principle [15]. It can not only simulate historical fires but also predict future fires; however, farsite, can only predict future fires for a short time. In addition, many types of data are required to simulate fires in farsite, and some of these data are difficult to obtain. CA [16-19] has been applied in many disciplines because it is easy to use on computers [20]. CA $[21,22]$ has better simulated performance in complex forest environments because fires do not usually spread in elliptical patterns. CA has been used by many scholars to simulate the spread of forest fires. O. Jellouli [23] used CA to simulate the spread of forest fires in the Oued Laou watershed (Morocco). Sun [24] produced accurate fire propagation maps by combining the CA framework with the Wang Zhengfei model at a fine scale. Zhou [25] proposed a spatial diffusion model based on multi-agent algorithm with CA, and Wang Zhengfei model is used as the ROS model. In these methods, the calculation and the transition rules of the cell's state need to be studied in depth.

Some new technologies have been used to simulate the fire spread to get a better simulation result, and machine learning based models have been in use for a long time [26,27]. Milanović [28] determined the main explanatory variables for forest fire occurrence for Logistic Regression (LR) and Random Forest (RF), and they mapped the probability of forest fire occurrence in Eastern Serbia based on these models. However, LR and RF models are more likely to produce under-fitting. Pimont [29] developed a Firelihood system, which is a two-component, Bayesian, hierarchically structured, probabilistic model of fire, but this approach has higher data requirements. With the development of neural networks, Zheng [30] realized fire spread, which used CA with extreme learning machine (ELM). According to historical fire data (vegetation, topography, and meteorological data), the probability of fire occurrence is calculated by ELM. In the probability model, the probability value of sample labels needs to be further studied. Hodges [31] proposed a machine learning approach to estimate the time-resolved spatial evolution of a wildland fire front using a deep convolutional inverse graphics network (DCIGN). Yang [32] proposed a CNN approach that uses remote sensing data to predict forest fires in Indonesia. Radke [33] proposed a new method of CNN-FireCast-which incorporated deep supervised machine learning methods in a unique model structure. The methods based on CNN are used to predict the spread of forest fires, in which the inputs are the images, and these methods increase the computational effort of the model.

Although the simulation can be carried out directly using transition rules with machine learning methods [34], it is difficult to use these methods to provide an ideal simulation result, because the sample data for training the parameters are hard to acquire. The ROS model remains the most important element for driving the propagation, and a key problem is how to estimate the ROS with higher precision [35]. Moreover, forest fire spread has a characteristic time dimension. Unlike CNN, RNN stores the previous information and applies it to the calculations of the current output. Forest fire spread methods that require fewer input data and can be driven by dynamic data in real time should be researched and applied quickly.

The proposed LSTM could be a good solution to the problem of gradient disappearance and the inability to predict long sequences of RNN; it has been proven to be an effective deep learning model in sequential tasks [36-38]. Many scholars have applied LSTM in many aspects and achieved good results. Qing [39] used LSTM to predict the weather and verified the superiority of LSTM. Wind plays a very important role in determining ROS, and fire can also generate changes in wind according to fire meteorology [40]. Wind is one of the most important influences on the ROS. The time series analysis of wind speed and 
the ROS can effectively improve the accuracy of ROS prediction. The method of predicting ROS by exploring the interaction between wind and fire needs to be investigated.

In this article, simulated methods of forest fire spread are studied with the accurate prediction of ROS and excellent performance of the propagation methods. The LSTM approach is used to predict ROS, and CA is used as the fire spread model. The previous wind speed and ROS are used as input into the LSTM to predict the ROS in the future. The S-LSTM model is designed according to the powerful interaction between wind and fire. The wind speed and the ROS are the inputs into the S-LSTM. At the same time, many models with the same structure are trained with the sampled data captured in different cases. LSTM-CA is designed to simulate the forest fire spread. Three correction rules, cosine correction, vegetation correction, and slope correction, are used to improve the simulation accuracy of the S-LSTM. In the end, three random wildfires are selected to validate the feasibility of the LSTM-CA model. The flowchart for simulating forest fire spread based on CA with LSTM as shown in Figure 1.



Figure 1. Flowchart for simulating forest fire spread based on CA with LSTM. Firstly, a fire spread rate model is designed and trained. Secondly, three kinds of fire rate correction methods are designed based on slope, slope direction, and vegetation, respectively. Finally, the model is verified with three wildfires, and the simulation result outperforms other models in the state of the art.

The rest of this paper is organized as follows. The ROS model based on an LSTM network (S-LSTM) is presented in Section 2. Section 3 designs the model of CA for the forest fire spread, and the correction rules are described in detail. Section 4 presents the methods of data collection and preprocessing. In Section 5, the experimental verification and experimental analysis are carried out by using data of three wildfires from remote sensing. Section 6 discusses the results and some prospects for future work.

\section{The Forest Fire Spread Model Based on LSTM}

\section{The Structure of the ROS Model}

In the process of forest fire spread, the ROS is changed because of the wind and other factors. Predicting the ROS with fewer and easier-to-measure data is an important aspect of fire fighting. 
The wind speed and the ROS change with time, and LSTM is a good method for predicting and analyzing time series. The one-dimensional matrices composed of the wind speed and the ROS are used as the input of normal LSTM. The structure of an LSTM is shown in Figure 2.

In Figure 2, $x^{t}$ is the input at time $t$ of the normal LSTM, $h^{t}$ is the output at time $t$, and the input and output are the wind speed and the ROS, respectively.

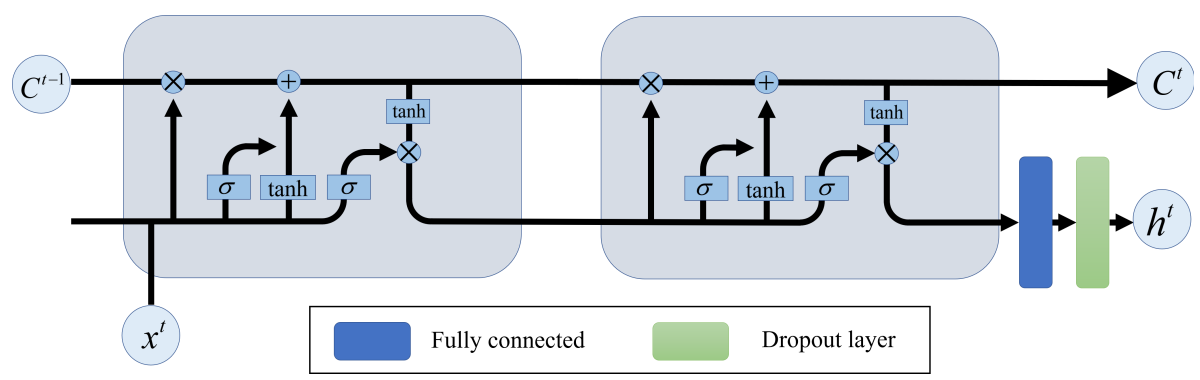

Figure 2. The LSTM cell structure. $x^{t}$ and $h^{t}$ are both one-dimensional matrices composed of wind speed and the ROS.

The wind plays an important role in the forest fire spread, at the same time, the fire will affect the local wind speed. From this result, the S-LSTM model is designed. In the structure of S-LSTM, the wind speed is used to control the forget gate and the ROS is used to control the input gate. In order to improve the fitting ability of the model, two LSTM units were set at each moment. The same unit of S-LSTM is used to record the changes in wind speed and the ROS. The structure of the S-LSTM model is shown in Figure 3.



Figure 3. The S-LSTM cell structure. The ROS $\left(V_{F}^{t}\right)$ and wind speed $\left(V_{W}^{t}\right)$ as input to the S-LSTM model, respectively.

In Figure $3, V_{W}^{t}$ and $V_{F}^{t}$ are the wind speed and the ROS at time $t . h_{W}^{t}, h_{F}^{t}$ are the prediction of the wind speed and the ROS at time $t . C^{t-1}$ and $C^{t}$ are the cell state at time $t-1$ and $t, \sigma$ is the sigmoid function [41], tanh is the hyperbolic tangent function [42]. These two functions are used as activation functions to solve problems that cannot be solved by linear models.

The wind speed and the ROS are used as input to S-LSTM, respectively. In Equations (1)-(4), the forget gate is used to detect the change in external wind speed and control the forget gate of the current unit state. According to the predicted output of the last unit state, the input gate controls the extent to which information is input to the unit. In the output gate, two control functions of output were set that can output the wind speed and the ROS speed, respectively. The models of normal LSTM and S-LSTM are compared in Section 5.1.

Forget gate:

$$
f^{t}=\sigma\left(W_{f}\left[\begin{array}{c}
V_{W}^{t} \\
h_{W}^{t-1}
\end{array}\right]+b_{f}\right)
$$


Input Gate:

$$
i^{t}=\sigma\left(W_{i}\left[\begin{array}{c}
V_{F}^{t} \\
h_{F}^{t-1}
\end{array}\right]+b_{i}\right)
$$

Update Cell State:

$$
C^{t}=f^{t} \cdot C^{t-1}+i^{t} \bullet \widetilde{C}^{t}
$$

Output Gate:

$$
\left\{\begin{array}{l}
o^{t}=\sigma\left(W_{o}\left[\begin{array}{c}
V_{W}^{t} \\
h_{W}^{t-1}
\end{array}\right]+b_{o}\right) \\
h_{W}^{t}=o^{t} \bullet \tanh \left(C^{t}\right) \\
o_{1}^{t}=\sigma\left(W_{o 1}\left[\begin{array}{c}
V_{F}^{t} \\
h_{F}^{t-1}
\end{array}\right]+b_{o 1}\right) \\
h_{F}^{t}=o^{t} \bullet \tanh \left(C^{t}\right)
\end{array}\right.
$$

\section{The Simulation of Forest Fire Spread Based on CA}

In Section 2, the ROS models are designed. In this section, fire spread is simulated by using CA with S-LSTM.

\subsection{The Simulated Logic Structure of Forest Fire Spread}

The CA model includes cell space, cell states, the type of neighborhood, and transformational rules. The scene of a fire is divided into cells, and physical quantities take on a finite set of values at each cell. Cells evolve according to a set of transition rules and the states of the adjacent cells in discrete time.

\subsubsection{The State Definition of CA}

The type of Moore neighborhood (Figure 4) is chosen to simulate two-dimensional fire spread. The $(i, j)$ denotes the cells at row $i$ and column $j$ in the whole-cell space.

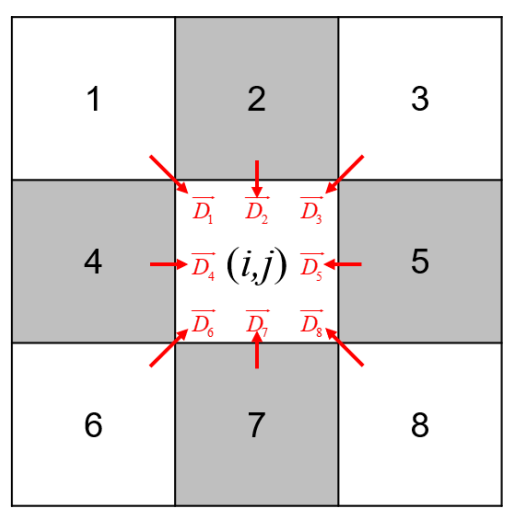

Figure 4. Moore-neighbourhood type of two-dimensional CA. The cells of 2, 4, 5, and 7 are adjacent cells. The cells of $1,3,6$, and 8 are sub-adjacent cells. $\overrightarrow{D_{i}}$ denotes the direction from the $i$-th cell to the central cell.

In the natural environment, the temperature of combustibles is lower than the ignition point. The temperature of combustible materials is raised to its ignition point due to external environmental factors. Then the combustible material begins to burn and transmit energy to its surroundings [43]. The cell states are classified into three types as follows.

State 1: the unburned state;

State 2: the early burn state, but the cell cannot spread to surrounding cells;

State 3: the completely burned state, whose cell can spread to surrounding cells. 
When the forest fire spread is simulated by CA, the state of cells at time $t$ is calculated according to the total area and the burned area of cell at the time $t$ [44].

$$
A_{i j}^{t}=\frac{S_{(i, j)}^{\prime}}{S_{(i, j)}}
$$

In Equation (5), the value range of $A_{i j}^{t}$ is $0 \leq A_{i j}^{t} \leq 1 . A_{i j}^{t}=0,0<A_{i j}^{t}<1$, and $A_{i j}^{t}=1$ represent the cells in state 1,2 , and 3 , respectively, at time $t$.

\subsubsection{The Simulation Model Structure of LSTM-CA}

Based on the fire spread data collected in the experimental area, a fire spread model using CA with S-LSTM is proposed. The framework of the LSTM-CA model is shown in Figure 5 .



Figure 5. The framework of the LSTM-CA model.

Where $W_{t-1}, F_{t-1}$ are the wind speed and the ROS at time $t-1$. The ROS $F_{t}$ at time $t$ is calculated from the S-LSTM. After cosine correction, existing vegetation canopy cover correction and slope direction correction are performed, and whether the state of the central cell changes depends on the effect of the surrounding cells on it. When the fire spreads between cells, the appropriate S-LSTM model is selected according to the slope, slope direction, wind speed, and wind direction.

\subsection{State Transformational Rules and Correction Methods}

CA has the ability to simulate the spatiotemporal evolution of complex systems, because the state transformational rules are determined by the current state of the cell and its adjacent cells. There are errors between the structured laboratory environment and the real environment. In the actual environment, the slope direction also determines the spread direction of the fire, and the fuel kind at each location is also different. Each cell is given the parameter of slope direction and existing vegetation coverage. The predicted results of S-LSTM are corrected.

\subsubsection{Transformational Rules}

Rule 1 states that a cell that is non-combustible will never burn. Rule 2 states that if a cell is in state 2 at time $t$, it will becomes state 3 at time $t+1$, but it cannot spread to its surrounding cells. Rule 3 states that if a cell is in state 3 at time $t$, the fire can spread to 
adjacent cells. Rule 4 states that if a cell is in state 1 at time $t$ and there are cells in state 3 in the adjacent cells, the cell will burn at time $t+1$.

\subsubsection{Cosine Correction for the Result of S-LSTM}

To minimize errors caused by laboratory experiments, the cosine similarity [45-48] is used to evaluate the similarity between the model and the actual parameters. The wind direction and slope values of the model form the vector $\left(\alpha_{w 1}, \alpha_{s 1}\right)$, and the actual values of wind direction and slope form the vector $\left(\alpha_{w 2}, \alpha_{s 2}\right)$. The formula for calculating the cosine similarity is shown in Equation (6).

$$
\cos \theta=\frac{\sum\left(\alpha_{w i} \cdot \alpha_{s i}\right)}{\sqrt{\sum \alpha_{w i}^{2}} \cdot \sqrt{\sum \alpha_{s i}^{2}}}
$$

In the fire spread, slope is one of the most variable parameters. Therefore, the difference in slope is used to measure the increase or decrease of the cosine correction. If the slope of the model is higher than the actual slope, the calculation method is shown in Equation (7); If the model slope is lower than the actual slope, the calculation method is shown in Equation (8).

$$
\begin{aligned}
& V_{c}=V_{L S T M}+V_{L S T M} *(1-\cos \theta) \\
& V_{c}=V_{L S T M}-V_{L S T M} *(1-\cos \theta)
\end{aligned}
$$

where $V_{c}$ represents the ROS after cosine correction. $V_{L S T M}$ represents the output of SLSTM, $\cos \theta$ represents cosine similarity between model parameters and real parameters, and $1-\cos \theta$ represents the cosine distance [49] between the $\left(\alpha_{w 1}, \alpha_{s 1}\right)$ and $\left(\alpha_{w 2}, \alpha_{s 2}\right)$.

\subsubsection{Correction Rules of Vegetation}

The ROS is different at different combustible loads [50,51]. The fuel is divided into three types-forest, shrub, and meadow-and each type has a different EVC. The ROS increases and then decreases with the increase of vegetation thickness. Therefore, according to this characteristic, each kind of fuel is mapped to the sine function from $0^{\circ}$ to $180^{\circ}$.

For example, the conversion formula of the EVC parameter is shown in Equation (9).

$$
e v c_{-} t=\frac{180-0}{199-110} \times(e v c-110)=2.02(e v c-110)
$$

where the value of EVC after mapping is $e v c_{-} t$. If the cells cannot burn, the regulation factor is -1 , which means that the cells will not be received and pass to the fire to spread. The algorithm of correction factors for different types and different vegetation coverage is as follows:

$$
K_{e v c}=1+\sin \left(E_{c}\right)-\sin \left(E_{n}\right)
$$

In Equation (10), $E_{c}, E_{n}$ is the EVC of the central cell and the adjacent cell after mapping.

The ROS is affected by the fuel load. According to the empirical model, the vegetation adjustment factor for the spread of fuel in the forest fire is designed. The value range of $K_{e v c}$ is $[0,2]$. The value range of $E_{c}, E_{n}$ is $[0,1)$, and vegetation factors will reduce the ROS. When the value is 1 , vegetation factors have no effect on the ROS. When the value is in the range of $(1,2]$, vegetation factors promote the ROS.

\subsubsection{Correction Rules of Slope Direction}

With the slope, the slope direction determines whether it is an up-slope or down-slope, so the slope direction plays a very important role in fire spread. The main direction vector $\overrightarrow{v_{\text {and }}}$ of fire spread is calculated by the wind vector $\overrightarrow{v_{\text {wind }}}$ and slope vector $\overrightarrow{v_{\text {slope }}}$, as shown in Equation (11).

$$
\overrightarrow{v_{\text {and }}}=\overrightarrow{v_{\text {wind }}}+\overrightarrow{v_{\text {slope }}}
$$


Wind direction is defined as the direction of wind blowing in meteorology, and wind direction and slope direction are based on normal north. When the slope direction and wind direction are the same as the fire spread direction, both of them have a restraining effect on the fire spread, so the opposite direction of the wind and slope are used to calculate the main direction of fire spread.

$$
\begin{gathered}
\vec{v}=v_{w s} \cdot \overrightarrow{v_{u}} \\
\vec{e}=\frac{\overrightarrow{A B}}{|A B|}
\end{gathered}
$$

The Min-Max Normalization method is used for the summation vector because the wind speed and the slope have different units. The slope and wind speed are normalized to $(0,10)$. Equation $(12)$ is used to calculate wind vector and slope vector, $v_{w s}$ indicates the normalized value of wind speed or slope, and $\overrightarrow{v_{u}}$ denotes the unit vector of the calculated wind direction and slope direction. The unit vector in the main direction of the spread is calculated by Equation (13).

$$
\vec{v}=v \cdot \overrightarrow{v_{\text {and }}}
$$

In Equation (14), $v$ is the ROS, which is predicted by the S-LSTM network. $\overrightarrow{v_{\text {and }}}$ is the $v e \vec{c}_{a n d}$ calculated by Equation (13). After the main direction is calculated, the unit vector of the spread direction from the surrounding cells to the central cell is also calculated. The unit vector $(\vec{S})$ from the surrounding cells to the central cell is shown in Figure 6. Details are shown in Table 1.

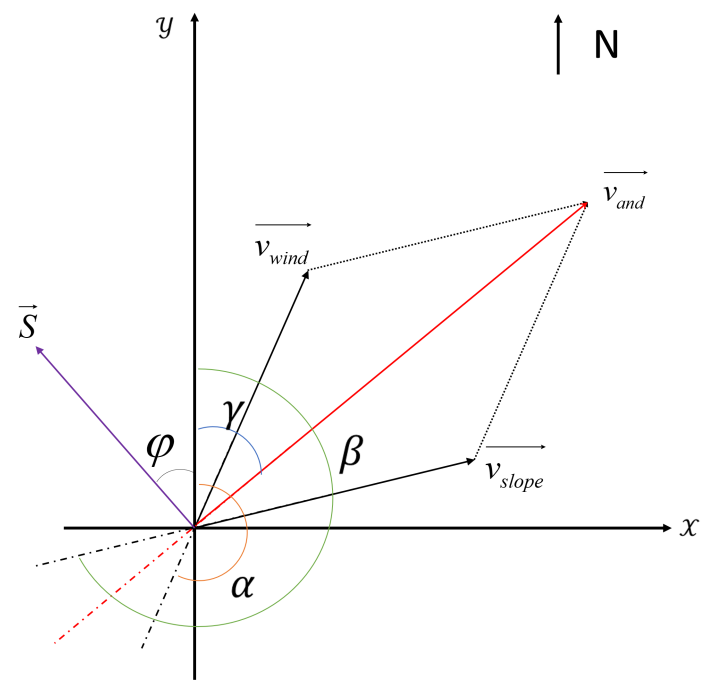

Figure 6. Wind direction and slope vector diagram. $\alpha$ is the angle of wind direction, $\beta$ is the angle of slope direction, $\gamma$ is the angle of main direction, and $\varphi$ is the angle between the spread direction $(\vec{S})$ and the positive direction of the y-axis.

The calculation method of the slope direction factor is shown in Equation (15):

$$
K_{\text {slope }}=1+\vec{v} \cdot \overrightarrow{S_{n}}, n \in[1,2,3,4,5,6,7,8]
$$

In this study, the vector sum of the slope direction and wind direction is used to analyze the impact of the fire spread. In the CA, the vector sum composed of adjacent cells and central cells is established to calculate the effect of fire spread in different directions. 
Table 1. Unit vectors formed by adjacent cell and central cell.

\begin{tabular}{ccc}
\hline The Spread of Vector & Mathematical Expression & Angle \\
\hline $\overrightarrow{S_{1}}$ & {$\left[\sin \varphi_{1}, \cos \varphi_{1}\right]$} & $\varphi_{1}=135^{\circ}$ \\
$\overrightarrow{S_{2}}$ & {$\left[\sin \varphi_{2}, \cos \varphi_{2}\right]$} & $\varphi_{2}=180^{\circ}$ \\
$\overrightarrow{S_{3}}$ & {$\left[\sin \varphi_{3}, \cos \varphi_{3}\right]$} & $\varphi_{3}=225^{\circ}$ \\
$\overrightarrow{S_{4}}$ & {$\left[\sin \varphi_{4}, \cos \varphi_{4}\right]$} & $\varphi_{4}=90^{\circ}$ \\
$\overrightarrow{S_{5}}$ & {$\left[\sin \varphi_{5}, \cos \varphi_{5}\right]$} & $\varphi_{5}=270^{\circ}$ \\
$\overrightarrow{S_{6}}$ & {$\left[\sin \varphi_{6}, \cos \varphi_{6}\right]$} & $\varphi_{6}=45^{\circ}$ \\
$\overrightarrow{S_{7}}$ & {$\left[\sin \varphi_{7}, \cos \varphi_{7}\right]$} & $\varphi_{7}=0^{\circ}$ \\
$\overrightarrow{S_{8}}$ & {$\left[\sin \varphi_{8}, \cos \varphi_{8}\right]$} & $\varphi_{8}=315^{\circ}$ \\
\hline
\end{tabular}

\subsection{Establishment of Forest Fire Spread Model}

If the cell is in the cumulative state at time $t$, it will become to completely burn state based on the state of itself and its adjacent cells.

$$
V=V_{c} \cdot K_{e v c} \cdot K_{\text {slope }}
$$

In Equation (16), $V$ represents the ROS from adjacent cell to the central cell, $V_{c}$ is the prediction of the S-LSTM model after cosine correction, $K_{e v c}$ is the vegetation influence factor, and $K_{\text {slope }}$ is the influence factor of the slope direction.

In this study, the real area of the unit cell grid is $30 \times 30 \mathrm{~m}^{2}$. Understanding how to calculate the combustion area of CA has become an important way of reducing the spread error. In the current research, the spread of forest fire has been verified and recognized by elliptical spread theory. A method to calculate the combustion area of cells is proposed. The unit grid cells continue to be divided into $1000 \times 1000$ squares. By calculating the number of squares, the area of cells is calculated. The spread method between cells is shown in Figure 7.

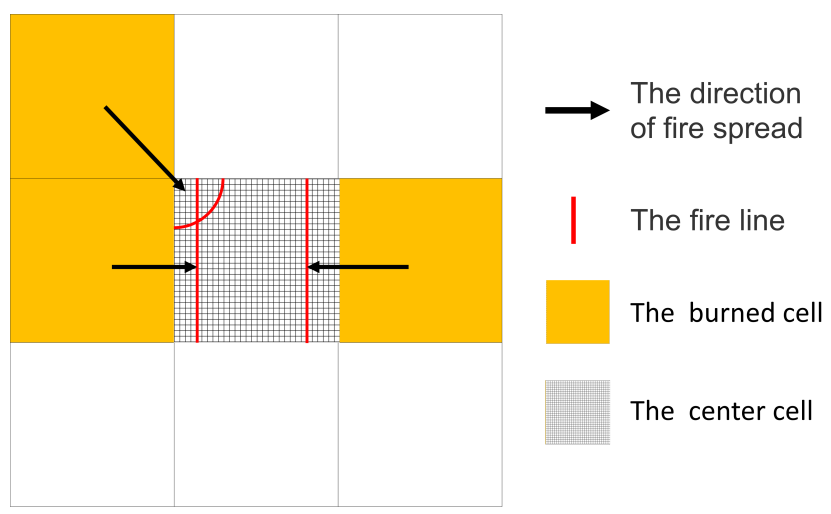

Figure 7. Example of fire spreading from the eight directional cells to the central cell. The adjacent cells spread to the central cell in a rectangle, and the sub-adjacent cells spread to the central cell in a quarter circle.

In Equation (5), $S_{(i, j)}^{\prime}$ is calculated as shown in Equation (17); $a$ represents the adjacent cell, $b$ represents the sub-adjacent cell.

$$
S_{(i, j)}^{\prime}=\sum V_{a} * \Delta t+\sum \pi *\left(V_{b} * \Delta t\right)^{2} / 4
$$




\section{Data Collection and Preprocessing}

\subsection{The Experimental Design}

The surface fuel is selected from Maoer mountain, Harbin, Heilongjiang Province, China, $45^{\circ} 24^{\prime} \mathrm{N}, 127^{\circ} 39^{\prime} \mathrm{E}$. At present, the forest landscape of the Maoershan is composed of natural secondary forests dominated by precious broad-leaved forest, poplar-birch forest, oak forest, and artificial forests such as Korean pine, larch, and Pinus sylvestris var.

In Figure 4, Moor-type CA is designed according to the two factors that have the greatest impact on fire spread, slope and wind, and fire spread experiments were conducted on this basis. Suppose the fire spreads in the direction $\overrightarrow{D_{7}}$ and the wind direction is $\overrightarrow{D_{1}}$ and $\overrightarrow{D_{3}}, \overrightarrow{D_{4}}$ and $\overrightarrow{D_{5}}, \overrightarrow{D_{6}}$ and $\overrightarrow{D_{8}}$ have the same effect on the spread of the fire. Five kinds of fire spread are designed according to the angle between fire spread direction and wind direction: $0^{\circ}, 45^{\circ}, 90^{\circ}, 135^{\circ}$, and $180^{\circ}$.

According to the different slopes, the three fire spreading scenarios, uphill, downhill, and flat slope, are studied separately. Therefore, 15 kinds of fire spread experiments were designed, as shown in Figure 8. Because the combustibles are collected from Maor Mountain with the maximum slope of $15^{\circ}$, the middle value $8^{\circ}$ is selected to facilitate the correction w.r.t. the slope in the stage of simulation using the trained model. In fact, the selected slope has little effect on the model, because the ROS model (S-LSTM) mainly considers the interaction between fire and wind, and the correction in the simulation can eliminate the error from slope selection here.

Figure 9 shows the experimental scene that is captured by an infrared camera, and the detailed experimental parameters are shown in Table 2.

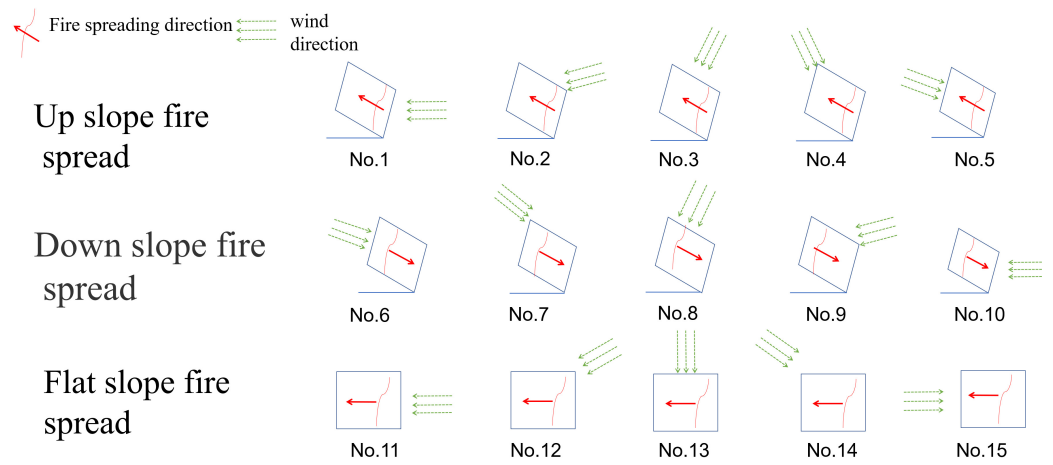

Figure 8. Fifteen kinds of fire spread experiments. Three types of slope. Five kinds angle between fire spread direction and wind direction. From left to right, the angle between the wind direction and fire spread direction is $0^{\circ}, 45^{\circ}, 90^{\circ}, 135^{\circ}$, and $180^{\circ}$.

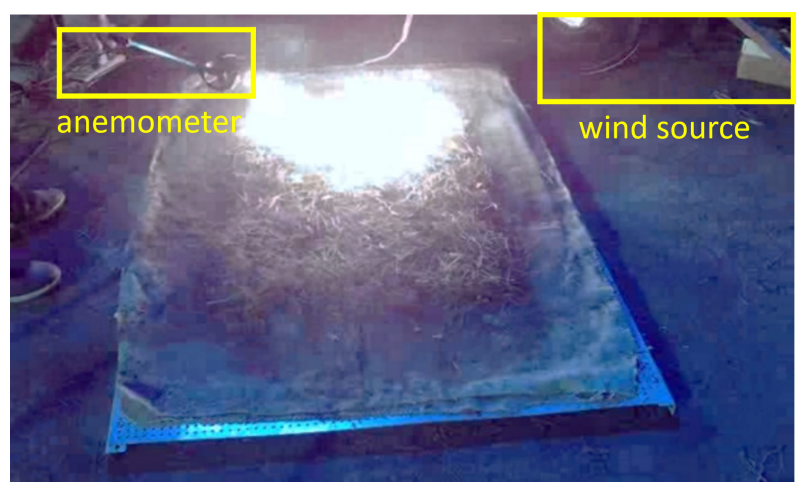

Figure 9. Burning experiment configuration. The picture shows a hand-held anemometer in the upper-left corner and a wind source in the upper-right corner. 
Table 2. Fifteen kinds of fire spread test parameters. Three types of slope and five kinds angle between fire spread direction and wind direction.

\begin{tabular}{cccc}
\hline NO. & Surface Area $\left(\mathbf{m}^{\mathbf{2}}\right)$ & Slope $\left(^{\circ}\right)$ & Angle between Wind Direction and Fire Spread Direction $\left(^{\circ}\right)$ \\
\hline 1 & $0.6 \times 0.8$ & Up slope $/ 8^{\circ}$ & $0^{\circ}$ \\
2 & $0.6 \times 0.8$ & Up slope $/ 8^{\circ}$ & $45^{\circ}$ \\
3 & $0.6 \times 0.8$ & Up slope $/ 8^{\circ}$ & $90^{\circ}$ \\
4 & $0.6 \times 0.8$ & Up slope $/ 8^{\circ}$ & $135^{\circ}$ \\
5 & $0.6 \times 0.8$ & Up slope $/ 8^{\circ}$ & $180^{\circ}$ \\
6 & $0.6 \times 0.8$ & Down slope $/ 8^{\circ}$ & $0^{\circ}$ \\
7 & $0.6 \times 0.8$ & Down slope $/ 8^{\circ}$ & $45^{\circ}$ \\
8 & $0.6 \times 0.8$ & Down slope $/ 8^{\circ}$ & $90^{\circ}$ \\
9 & $0.6 \times 0.8$ & Down slope $/ 8^{\circ}$ & $135^{\circ}$ \\
10 & $0.6 \times 0.8$ & Down slope $/ 8^{\circ}$ & $180^{\circ}$ \\
11 & $0.6 \times 0.8$ & Flat slope $/ 0^{\circ}$ & $0^{\circ}$ \\
12 & $0.6 \times 0.8$ & Flat slope $/ 0^{\circ}$ & $45^{\circ}$ \\
13 & $0.6 \times 0.8$ & Flat slope $/ 0^{\circ}$ & $90^{\circ}$ \\
14 & $0.6 \times 0.8$ & Flat slope $/ 0^{\circ}$ & $135^{\circ}$ \\
15 & $0.6 \times 0.8$ & Flat slope $/ 0^{\circ}$ & $180^{\circ}$ \\
\hline
\end{tabular}

\subsection{Calculating the ROS}

The ROS is calculated by the distance and time between the fire points on the two adjacent infrared images [52]. The angle between the camera and the combustion bed causes rectangles in space to become irregular shapes in the image. The perspective transformation [53-55] transforms the original image into an Orthographic projection image in which the corresponding actual distances between points in the image can be solved. Four calibration points with temperatures higher than the surrounding ones are placed on the same surface of the rectangular combustion bed, which is used to calculate the perspective transformation matrix of the image.

The perspective transformation matrix is calculated through the calibration points in Figure 10a, which are used for perspective transformation in the fire spread. Figure $10 \mathrm{~b}$ shows the image after perspective transformation. Then, the noise in the image is removed by the median filter. After preprocessing, the fire line at each moment is obtained, and the actual spread distance can be calculated. The fire line is depicted in Figure 10c.

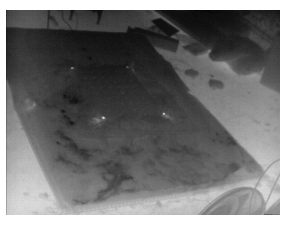

(a)



(b)

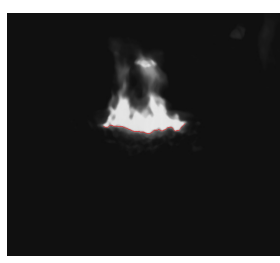

(c)

Figure 10. The extraction process of the infrared image fire line. (a) is an infrared image with four calibration points, (b) is the image after perspective transformation, and (c) is the infrared image with the fire line.

$$
\begin{aligned}
& {\left[\begin{array}{l}
X \\
Y \\
Z
\end{array}\right]=\left[\begin{array}{lll}
a_{11} & a_{12} & a_{13} \\
a_{21} & a_{22} & a_{23} \\
a_{31} & a_{32} & a_{33}
\end{array}\right]\left[\begin{array}{l}
x \\
y \\
z
\end{array}\right]} \\
& \left\{\begin{array}{l}
X^{\prime}=\frac{X}{Z}=\frac{a_{11} x^{\prime}+a_{12} y^{\prime}+a_{13}}{a_{31} x^{\prime}+a_{32} y^{\prime}+a_{33}} \\
Y^{\prime}=\frac{Y}{Z}=\frac{a_{21} x^{\prime}+a_{22} y^{\prime}+a_{23}}{a_{31} x^{\prime}+a_{32} y^{\prime}+a_{33}} \\
Z^{\prime}=\frac{Z}{Z}=1
\end{array}\right.
\end{aligned}
$$


In Equation (18), $X, Y, Z$ represent the geospatial coordinates of the four points. $x, y$ are pixel coordinates of infrared image, and $z$ is depth scaling factor. a $3 \times 3$ perspective transformation matrix can be obtained by the four pixel coordinates and geographic coordinates. $X^{\prime}, Y^{\prime}$ is the pixel coordinate of the image after the perspective transformation.

\section{Results and Discussion}

\subsection{The ROS Prediction of S-LSTM Compared with Normal LSTM}

The design of the LSTM and S-LSTM was presented in Section 2, and wind speed and the ROS are input into the LSTM for the ROS prediction. In the S-LSTM model, wind speed influences the change of the ROS through the forget gate. The ROS at the moment $t+1$ is predicted according to $t-4, t-3, t-2, t-1$, and $t$. Seventy percent of the experimental data in each group form the training set, twenty percent form the validation set, and ten percent form the test set. In order to ensure that the predictions of the LSTM and S-LSTM are comparable, the same hyper-parameters are used. Before training models, multiple groups of hyper-parameters are set. The optimal set of models' hyper-parameters is shown in Table 3.

Table 3. The hyperparameters for training the LSTM-based model (S-LSTM).

\begin{tabular}{cccccc}
\hline LSTM Layers & Learning Rate & Units & Batch Size & Time Step & Iterations \\
\hline 2 & 0.001 & 10 & 15 & 5 & 1200 \\
\hline
\end{tabular}

In order to obtain a reliable and stable model, cross validation is adopted. Cross validation is a method to estimate the general ability of models in the field of statistics and machine learning. Cross validation can solve the problem of over-fitting and underfitting. The training set is divided into five sample sets, and the model is trained five times. During each training, one of the data sets is used as a test set, and the other four are used as training sets. The 5-fold cross-validation method is shown in Figure 11.

\begin{tabular}{|l|l|l|l|l|l|l|}
\hline \multicolumn{7}{|c|}{ Training Dataset } \\
\hline Fold 1 & Fold 2 & Fold 3 & Fold 4 & Fold 5 \\
\hline & Training set & & Test set & \\
\hline Fold 1 & Fold 2 & Fold 3 & Fold 4 & Fold 5 & Result 1 \\
\hline Fold 1 & Fold 2 & Fold 3 & Fold 5 & Fold 4 & Result 2 \\
\hline Fold 1 & Fold 2 & Fold 4 & Fold 5 & Fold 3 & Result 3 \\
\hline Fold 1 & Fold 3 & Fold 4 & Fold 5 & Fold 2 & Result \\
\hline Fold 2 & Fold 3 & Fold 4 & Fold 5 & Fold 1 & Result 4 \\
\hline
\end{tabular}

Figure 11. Five-Fold cross Validation method. A total of $4 / 5$ of the training data are used to train the model, $1 / 5$ of the data are used for prediction, and the errors of the five prediction are averaged.

The performance of models can be judged by the loss curve. The training loss and validation loss curves of LSTM and S-LSTM model for 15 kinds of experiments are shown in Figure 12. From Figure 12, it is concluded that neither LSTM nor S-LSTM models produce over-fitting or under-fitting. For the same training set and validation set, the convergence speed and loss value of S-LSTM model are lower than that of the normal LSTM model. 




(a) No. 1

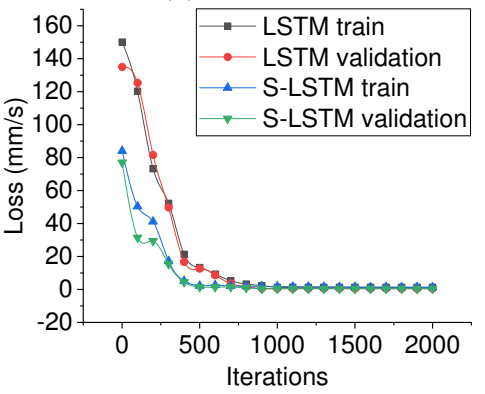

(d) No. 4

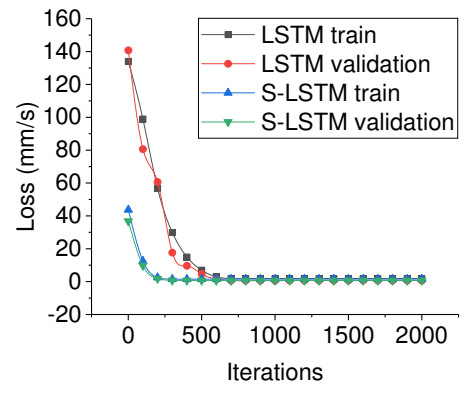

(g) No. 7

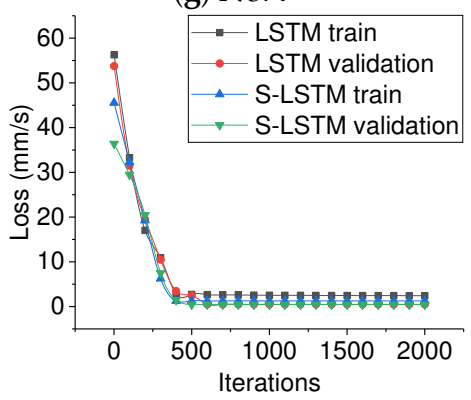

(j) No. 10

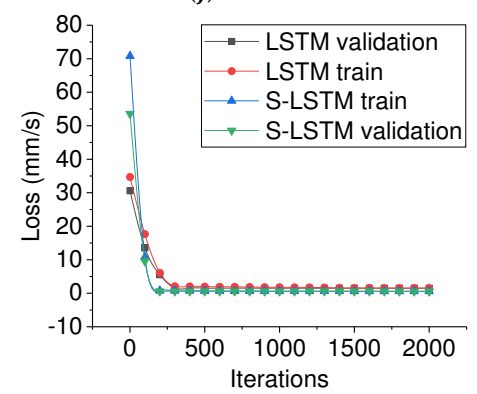

(m) No. 13

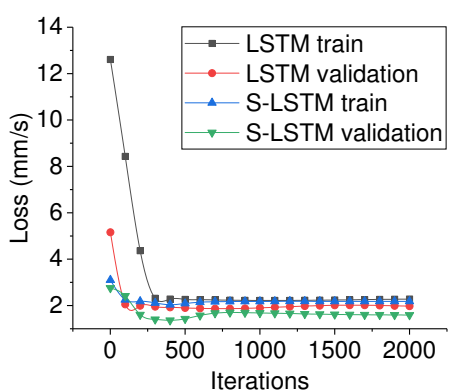

(b) No. 2



(e) No. 5

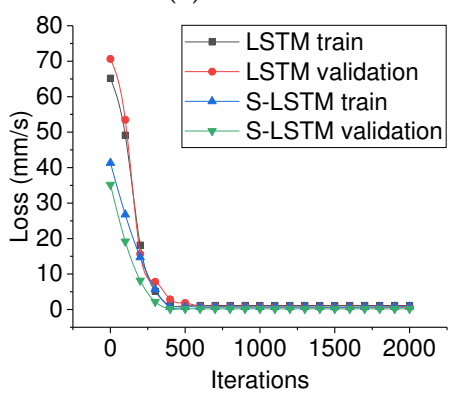

(h) No. 8

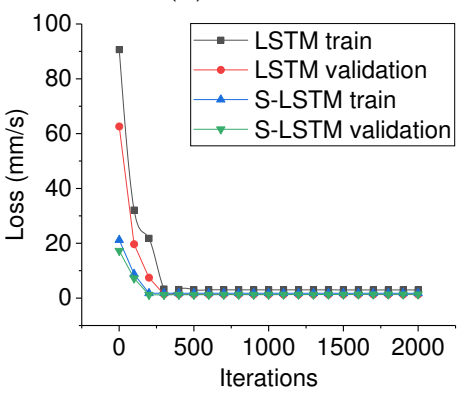

(k) No. 11



(n) No. 14



(c) No. 3



(f) No. 6

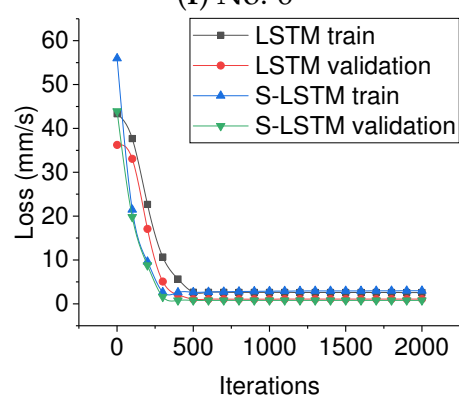

(i) No. 9



(1) No. 12

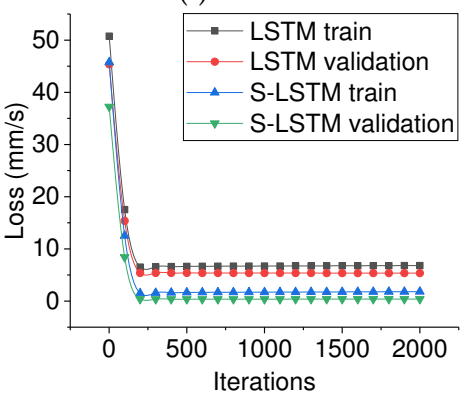

(o) No. 15

Figure 12. The train loss and validation loss for both the normal LSTM and S-LSTM based on the dataset collected with the 15 kinds of configurations shown in Table 2. The subfigures (a-o) are related to the data slots of No. (1-15) in Table 2, respectively. 
Mean-Square Error (MSE) [56-59] is a measure that reflects the difference between the prediction and real values. It is used to evaluate the prediction performance of normal LSTM and S-LSTM.

$$
M S E=\frac{1}{n} \sum_{i=1}^{n}\left(\hat{y}_{i}-y_{i}\right)^{2}
$$

Fifteen groups of fire spread experiments are limited by the experimental site, and the number of data collected is different. In Equation (20), $n$ is $10 \%$ of the total experimental data in each group. $\hat{y}_{i}$ is the predicted ROS, and $y_{i}$ is the true ROS. The MSE of the two LSTM structures in 15 types of fire spread is shown in Figure 13.



Figure 13. The MSE of predicting the fire spread rate for both the normal LSTM and the S-LSTM based on the dataset collected with the 15 kinds of configurations shown in Table 2. The histograms No. 1-15 are related to he data slots of No. (1-15) in Table 2, respectively.

In this paper, the same S-LSTM model is applied to the training and prediction of 15 fire spread experiments. S-LSTM only reflects the interaction between wind and fire, and other factors affecting the fire spread are not considered. In addition, there are some errors in the process of data collection. The above causes inconsistent model predictions for 15 types of fire spread data, and even the error of the two groups of data is slightly higher than normal LSTM.

\subsection{The Fire Region Position Prediction of LSTM-CA Compared with ELM-CA}

\subsubsection{The Study Areas}

The remote sensing image data of forest fires were collected from LANDFIRE [60], MTBS and other websites. The data were used to analyze the applicability of LSTM-CA and ELM-CA. The information on three wildfires is shown in Figure 14. According to the model input requirements, the slope, slope direction, vegetation type, vegetation height, vegetation coverage, and elevation files were downloaded. The resolution of the remote sensing image was $30 \mathrm{~m}$. This ensures that the pre-fire vegetation parameter of each fire is persuasive. 


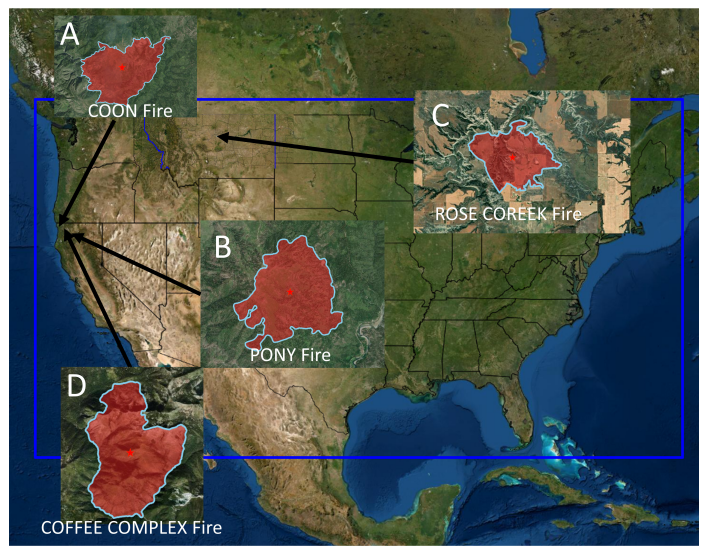

Figure 14. The four wildfires that happend in the United States (the red stars show the starting point of each fire).

The COON wildfire broke out on 1 August 2015. It was the first wildfire of the simulation object. This fire was located $36.5 \mathrm{~km}$ southwest of Reading City, California. The burned area is about $24.23 \mathrm{~km}^{2}$ and the altitude of the selected region is about $65-910 \mathrm{~m}$. The monthly average temperature was $18.5^{\circ} \mathrm{C}$, the average humidity was $52 \%$, and the monthly precipitation was $50.04 \mathrm{~mm}$.

The PONY wildfire broke out on 7 June 2016. It is the second wildfire of the simulation object. It was located $17.54 \mathrm{~km}$ southwest of Camp Hapi, California. The burned area is about $12.6 \mathrm{~km}^{2}$, and the altitude of the selected region is about 236 to $1668 \mathrm{~m}$. The monthly average temperature was $15.82{ }^{\circ} \mathrm{C}$, the monthly average humidity was $55.18 \%$, and the monthly precipitation is $12.12 \mathrm{~mm}$.

The ROSE CREEK wildfire broke out on 3 September 2018. It was the third wildfire of the simulation object. The fire was located $67.4 \mathrm{~km}$ west of Chautto, Montana. The burned area was about $4.57 \mathrm{~km}^{2}$, and the altitude of the selected region is about 887 to $1026 \mathrm{~m}$. The monthly average temperature was $18.06^{\circ} \mathrm{C}$, the monthly average humidity was $46.17 \%$, and the monthly precipitation was $5.28 \mathrm{~mm}$.

The COFFEE COMPLEX wildfire broke out on 2 August 2014, located $41.9 \mathrm{~km}$ northeast of Waverville, California. This wildfire is used to train the ELM. The 7000 sample points from the burned area and the 7000 sample points from the unburn area are selected as the training data of ELM. The burned area was about $25.9 \mathrm{~km}^{2}$, and the altitude is about 974 to $2346 \mathrm{~m}$. The average monthly temperature was $23.97^{\circ} \mathrm{C}$, the average humidity was $37.27 \%$, and the monthly precipitation was $8.32 \mathrm{~mm}$.

\subsubsection{The Results of LSTM-CA and ELM-CA}

The first step in simulating the spread of forest fire is to analyze the topography and environment of the burning area. Secondly, the main direction of fire spread is determined, and the appropriate S-LSTM model is selected. Then, the results of the S-LSTM model are modified according to the correction rules proposed in Section 3. According to the transition rules, the cell state is transformed and the forest fire spread simulation is completed.

In order to verify the effectiveness of the model, this paper sets up two comparative experiments: the first experiment compares the simulation results with the actual results, and the second experiment compares the results of LSTM-CA and ELM-CA. ELM-CA [61] trains the ELM model by collecting information about combustion and unburned areas and recording wind speed in the event of a fire. The probability is predicted by the action of elm and wind. Fourteen thousand samples were randomly selected from the COFFEE COMPLEX fire. The burnt point is marked as 1 , and the unburned point is marked as 0 . Each cell has corresponding slope, aspect, vegetation elevation, vegetation cover, vegetation type, and elevation information as input to the ELM model.

In Figures 15-17, the simulation results of LSTM-CA and ELM-CA for the same three wildfires are shown. The curves of different colors represent the actual fire areas of three 
wildfires, and the red area represents the LSTM-CA and ELM-CA simulations. There is a lack of burning time data when collecting the fire information from LANDFIRE and other websites. In this study, fire information was imported into Farsite for simulation, and the burning time of the three wildfires was obtained. COON, PONY, and ROSE COREAK simulated times were $128 \mathrm{~h}, 150 \mathrm{~h}$, and $80 \mathrm{~h}$, respectively. When simulating three wildfires, the interval of each iteration was $1 \mathrm{~h}$, so the simulation of three wildfires was iterated 128 times, 150 times, and 80 times, respectively.

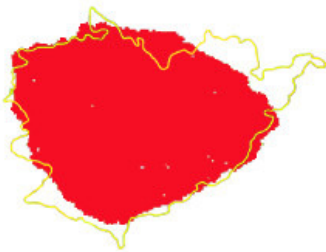

(a)



(b)

Figure 15. The prediction and real burned region of COON wildfire. (a) The comparison of burned regions between LSTM-CA prediction and reality. (b) The comparison of burned regions between ELM-CA prediction and reality.



(a)

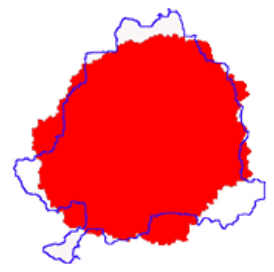

(b)

Figure 16. The prediction and real burned region of PONY wildfire. (a) The comparison of burned regions between LSTM-CA prediction and reality. (b) The comparison of burned regions between ELM-CA prediction and reality.

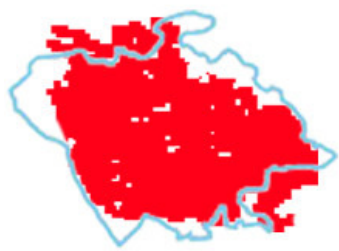

(a)

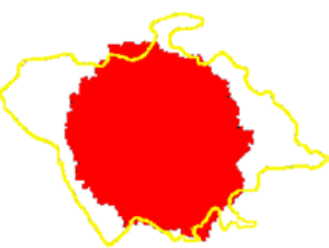

(b)

Figure 17. The predicted and real burned regions of ROSE COREAK wildfire. (a) The comparison of burned regions between LSTM-CA prediction and reality. (b) The comparison of burned regions between ELM-CA prediction and reality.

\subsubsection{The Accuracy Analysis of Simulated Wildfire}

In order to compare the accuracy of the ELM-CA model and the LSTM-CA model, confusion matrix [62-65] and Hausdorff distance [66] were selected as two evaluation methods [67]. The confusion matrix is a specific matrix used to visualize the performance of models. There are two ways to evaluate the model based on the confusion matrix: one is the KAPPA coefficient [68] and the other is the commission and omission error. Hausdorff distance can effectively characterize the similarity of the edge contour between objects.

(1) The confusion matrix analysis of LSTM-CA and ELM-CA

In the real burned results and simulated results, the pixels in the image can be divided into two categories: the burned pixels and the unburned pixels. According to the confusion 
matrix, the following four sample concepts are established: the simulated burning area is the real burning area (true positive). The simulated burned area is the real unburned area (false positive). The simulated unburned area is the real burning area (false negative). The simulated unburned area is the real unburned area (true negative). Tables 4 and 5 show the percentages of three types of fires simulated by LSTM-CA and ELM-CA.

Table 4. The confusion matrix values of simulation results of the LSTM-CA model for three wildfires.

\begin{tabular}{ccccc}
\hline & True Positive (TP) & False Positive (FP) & False Negative (FN) & True Negative (TN) \\
\hline PONY & $28.5 \%$ & $5.1 \%$ & $5.5 \%$ & $60.9 \%$ \\
COON & $24.0 \%$ & $1.0 \%$ & $2.0 \%$ & $73.0 \%$ \\
ROSE CREEK & $31.2 \%$ & $5.0 \%$ & $1.4 \%$ & $62.4 \%$ \\
\hline
\end{tabular}

Table 5. The confusion matrix values of simulation results of the ELM-CA model for three wildfires.

\begin{tabular}{ccccc}
\hline & True Positive (TP) & False Positive (FP) & False Negative (FN) & True Negative (TN) \\
\hline PONY & $37.7 \%$ & $0.6 \%$ & $17.1 \%$ & $44.6 \%$ \\
COON & $25.2 \%$ & $0.7 \%$ & $8.2 \%$ & $65.9 \%$ \\
ROSE CREEK & $29.3 \%$ & $7.2 \%$ & $1.3 \%$ & $62.2 \%$ \\
\hline
\end{tabular}

Commission error is the percentage of the classification results that are misclassified, and omission error is the percentage of the classification results missing the real results. The formula is shown in Equation (21).

$$
\left\{\begin{array}{l}
C_{e}=1-\frac{T P}{T P+F P} \\
O_{e}=1-\frac{T P}{T P+F N}
\end{array}\right.
$$

In Equation (21), $C_{e}$ is the commission error and $O_{e}$ is the omission error. The commission error and omission error of the LSTM-CA and ELM-CA for the three wildfires are shown in Figure 18.

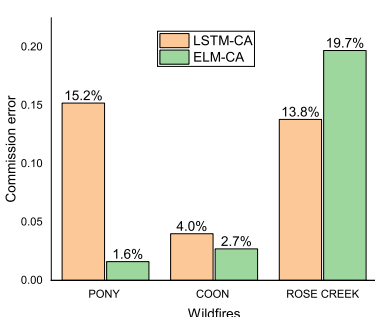

(a)

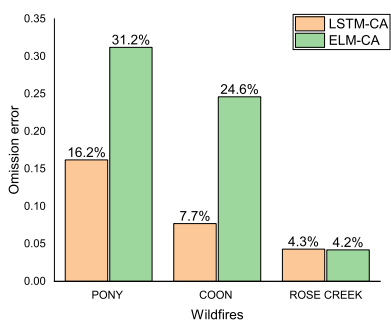

(b)

Figure 18. The commission error and omission error for three wildfires simulated by models LSTMCA and ELM-CA. (a) represents the commission error simulated by the two models. (b) represents the omission error simulated by the two models.

From Figure 18, for three randomly selected wildfires, there are different simulation performances for two models. The commission error of the PONY and COON wildfires simulated by LSTM-CA are higher than those of ELM-CA, especially the misclassification error of PONY, which reaches $15.2 \%$, but the omission errors of these two fires simulated by LSTM-CA are lower than those of ELM-CA.

KAPPA coefficient is used to evaluate the consistency $[69,70]$ between the simulated results and the real burned results.

$$
\left\{\begin{array}{l}
P_{0}=\frac{T N+T P}{T N+F P+F N+T P} \\
P_{e}=\frac{(T N+F P) *(T N+F N)+(F P+T P) *(F N+T P)}{(T N+F P+F N+T P)^{2}} \\
K=\frac{P_{0}-P_{e}}{1-P_{e}}
\end{array}\right.
$$


$P_{0}$ is observed consistency, and $P_{e}$ is expected consistency. $K$ is the KAPPA coefficient. According to Equation (22), the results are obtained as shown in Tables 6 and 7.

Table 6. The KAPPA coefficient of the LSTM-CA model's simulation results of three wildfires.

\begin{tabular}{cccc}
\hline & PONY & COON & ROSE CREEK \\
\hline$P_{0}$ & 0.89 & 0.97 & 0.94 \\
$P_{e}$ & 0.55 & 0.62 & 0.55 \\
$K$ & 0.76 & 0.92 & 0.86 \\
\hline
\end{tabular}

Table 7. The KAPPA coefficient of the ELM-CA model's simulation results of three wildfires.

\begin{tabular}{cccc}
\hline & PONY & COON & ROSE CREEK \\
\hline$P_{0}$ & 0.82 & 0.91 & 0.92 \\
$P_{e}$ & 0.49 & 0.58 & 0.55 \\
$K$ & 0.65 & 0.79 & 0.81 \\
\hline
\end{tabular}

The result of Kappa calculation is $[-1,1]$. However, it can be divided into five groups to represent different levels of consistency: $0.0 \sim 0.20$ for very low consistency, $0.21 \sim 0.40$ for general consistency, $0.41 \sim 0.60$ for moderate consistency, $0.61 \sim 0.80$ for high consistency, and $0.81 \sim 1$ for almost complete consistency. From Tables 6 and 7, it can be seen that the observation consistency, expected consistency, and KAPPA coefficient of LSTM-CA in the three wildfire simulations are better than ELM-CA.

In Figure 18, the KAPPA coefficients of LSTM-CA and ELM-CA are greater than 0.61, and the results are highly consistent. Analyzing a single fire shows that the LSTM-CA simulation results of the COON and ROSE CREEK wildfire are almost completely consistent with the results. The simulation results of the PONY wildfire are also highly consistent with the real results, and among the results of the three wildfires simulations by ELM-CA, only the ROSE CREEK wildfire just reached the almost consistent level, and the other two wildfires are only at a high level of consistency. Compared with ELM-CA, the accuracy of the LSTM-CA model is improved by $16.8 \%, 16.8 \%$, and $5.9 \%$ in the analysis of the three wildfires, PONY, COON, and ROSE CREEK.

(2) The Hausdorff distance analysis of LSTM-CA and ELM-CA

Hausdorff distance can effectively characterize the similarity of edge contours between objects and quantify the matching degree of edge contours. The Hausdorff distance is used to analyze the contour of predict results and real results.

The point set of predicted result contour in Euclidean space is $A=\left\{a_{1}, a_{2}, a_{3}, a_{4}, \ldots\right\}$. The point set of the real result contour in Euclidean space is $B=\left\{b_{1}, b_{2}, b_{3}, b_{4}, \ldots\right\}$. The Hausdorff distance is used to measure the distance between the two point sets. The smaller the Hausdorff distance value, the more similar the two point sets are.

$$
\left\{\begin{array}{l}
H(A, B)=\max [h(A, B), h(B, A)] \\
h(A, B)=\max _{a \in A} \min _{b \in B}\|a-b\| \\
h(B, A)=\max _{b \in B} \min _{a \in A}\|b-a\|
\end{array}\right.
$$

The Hausdorff distance between the simulation results and the actual combustion zone calculated by Equation (23) is as follows.

In Figure 19, the Hausdorff distances of the three wildfires simulated by LSTM-CA are all smaller than those of the simulation results of ELM-CA. Therefore, the contours of the LSTM-CA simulation results for the three wildfires are more similar to the real results. 


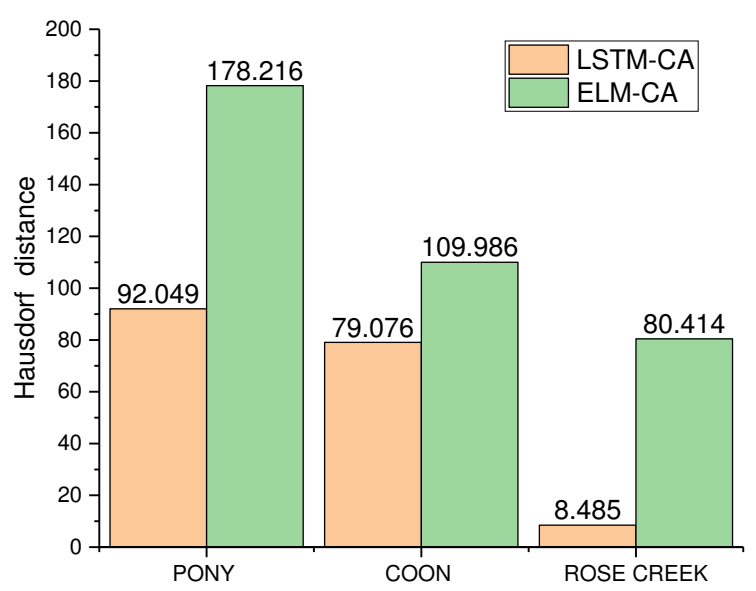

Figure 19. Comparison of Hausdorff distance between simulated and actual results of the two models.

\subsection{Discussion}

In the normal LSTM model, the wind speed and the ROS are simultaneously input to the input gate, output gate, and forget gate of the LSTM unit. The interaction between wind and fire is reflected through the network connection in the LSTM unit. In the S-LSTM model, wind speed controls the forget gate of the LSTM, and the interaction between wind and fire is more intense. The interaction between wind and fire is not only reflected by the neural network connection, which is more suitable for the fire spread in the actual environment; in addition, from the training loss and validation loss curves, as well as the MSE analysis of the test set, it can be concluded that S-LSTM has faster convergence performance and better fitting performance.

The superior performance of the model cannot be analyzed by the commission error and omission error; it is obvious that the LSTM-CA model has a significant advantage over the ELM-CA based on the KAPPA coefficient and the Hausdorff distance. This is because the slope direction is taken into account in the LSTM-CA, and the interaction between wind and fire is taken into account in the S-LSTM. However, the results differ based on whether it is the S-LSTM prediction for the 15 kinds experiments or the LSTM-CA and ELM-CA simulations for the three wildfires. This can be caused by the following.

For the S-LSTM, there are some errors in the data of the hand-held anemometer when it collects wind speed data. In addition, there is a difference between the fire line extracted from the infrared image and the actual fire line.

For the ELM-CA, (1) fire points are sampled from the burning area and the unburned area of the same fire. The sampled points are only collected after the end of a fire, so the data are not enough to train the ELM well. When the fire training model is applied to simulate the spread of another fire, the related properties of the fire area change, which reduces the prediction accuracy of the model. (2) When the state transition probability of a cell is higher than a random probability threshold, the cell can burn.

For the LSTM-CA, (1) the training data set comes from the laboratory experiments, and there are differences between laboratory experiments and real wildfires, so the trained model performs differently for the different real fires. (2) The cosine correction, vegetation correction, and slope correction of fire velocity cannot be completely consistent with the real value.

In addition, due to the distance between the weather station used to measure meteorological factors and the fire line, as well as the fact that the fire spread process can be influenced by human-made factors, rainfall, and other factors, there are some differences between the simulated results and the actual burning contours.

\section{Conclusions}

In order to quickly design risk management and effectively implement fire suppression policies, accurate prediction of the fire spread process is an important measure. Two ROS 
models are presented in this paper: the normal LSTM with a one-dimensional matrix consisting of wind speed and ROS as inputs, and the S-LSTM with wind speed and ROS as inputs. The performance of the S-LSTM model for predicting the ROS is verified by two methods. The first is to analyze the training loss and validation loss curves: the SLSTM is reduced to the lowest value within 500 training cycles for 15 kinds of experiments, with better convergence speed and validation loss values than the normal LSTM. The second is to analyze the mean square error of the model's predicted values for the test set: in the analysis of the 15 test sets, 2/3 of the mean squared errors were less than 1 and the other maximum mean squared errors were 2.73. The LSTM-CA model combining the S-LSTM and the CA was used to simulate forest fire spread, and three correction methods were designed based on vegetation, slope, and slope direction. The LSTM-CA model was shown to have a higher accuracy and was feasible in simulating forest fire spread by simulating and analyzing three real wildfires in the United States with the ELM-CA model. Based on the analysis of the KAPPA coefficients, there is a high consistency between the simulated and actual result of the LSTM-CA model, and the simulated results of the two fire can achieve almost complete consistency, while the simulated accuracy also reaches high consistency, in the fire whose simulated result was poor. In the analysis of Hausdorff distance, the ROSE CREEK wildfire was only 8.48 and the contours of the simulated results were similar to the actual results, the accuracy of the other two fires was acceptable. Due to the presence of errors, the commission errors of the LSTM-CA model are unsatisfactory compared with the ELM-CA model.

In addition, the process of forest fire spread is very complex, and the ROS is influenced by many factors, and the influence of various factors on fire spread needs to be studied in depth in future research.

Author Contributions: Conceptualization, X.L.; Data curation, X.L.; Formal analysis, T.H.; Investigation, L.S.; Methodology, M.Z.; Project administration, L.S.; Resources, J.L.; Software, S.Z.; Supervision, T.H.; Validation, S.S.; Visualization, S.S.; Writing—original draft, M.Z.; Writing—review and editing, X.L. All authors have read and agreed to the published version of the manuscript.

Funding: This work was supported by Natural Science Foundation of Heilongjiang Province of China (Grant No. LH2020C042); National Key Research and Development Program of China (Grant No. 2020YFC1511603); Fundamental Research Funds for the Central Universities (Grant No. 2572019CP20).

Institutional Review Board Statement: Not applicable.

Informed Consent Statement: Not applicable.

Data Availability Statement: Not applicable.

Acknowledgments: The author thanks the Northern Forest Fire Management Key Laboratory of the State Forestry and Grassland Bureau for providing the necessary devices for the experiment of this paper. The author would also like to thank editors and anonymous reviewers for their valuable suggestions to improve the quality of the manuscript.

Conflicts of Interest: The authors declare no conflict of interest.

\section{References}

1. Hu, T.-X.; Zhao, B.-Q.; Li, F.; Dou, X.; Hu, H.-Q.; Sun, L. Effects of fire on soil respiration and its components in a Dahurian larch (Larix gmelinii) forest in northeast China: Implications for forest ecosystem carbon cycling. Geoderma 2021, $402,115273$. [CrossRef]

2. Valente, F.; Laurini, M. Spatio-temporal analysis of fire occurrence in Australia. Stoch. Environ. Res. Risk Assess. 2021, 35, 1759-1770. [CrossRef]

3. Wang, W.; Zhang, X.-L.; Dou, X.; Li, F. Effect of Moderate Fire Disturbance on Soil Phosphorus and Potassium of Dahurian Larch (Larxi gmelinii) Forest. For. Eng. 2020, 36, 10-18.

4. Rossa, C.; Fernandes, P. Live fuel moisture content: The 'pea under the mattress' of fire spread rate modeling? Fire 2018, 1, 43. [CrossRef] 
5. Ahmad, A.D.; Abubaker, A.M.; Salaimeh, A.; Akafuah, N.K.; Finney, M.; Forthofer, J.M.; Saito, K. Ignition and burning mechanisms of live spruce needles. Fuel 2021, 304, 121371. [CrossRef]

6. Aleksandra, V.; Andrey, K.; Mikhail, K.; Tatiana, S. Fire Behavior Prediction in Larch Forests of the Kazakhstan Altai. Symmetry 2021, 13, 578.

7. Fernando, J.-P.-P.; Paula, T.-T.; Carmen, C.-R.; Jose, E.-M.L.; Alfonso, G.-F.; Francisco, J.-M.-C. Machine Learning Methods and Synthetic Data Generation to Predict Large Wildfires. Sensors 2021, 21, 3694.

8. Liang, H.; Zhang, M.; Wang, H.-L. A Neural Network Model for Wildfire Scale Prediction Using Meteorological Factors. IEEE Access 2019, 7, 176746-176755. [CrossRef]

9. Song, Y.-J.; Wang, Y.-H. Global Wildfire Outlook Forecast with Neural Networks. Remote Sens. 2020, 12, 2246. [CrossRef]

10. Banerjee, P. MODIS-FIRMS and ground-truthing-based wildfire likelihood mapping of Sikkim Himalaya using machine learning algorithms. Nat. Hazards 2021, 8, 1-37. [CrossRef]

11. Gao, C.; Lin, H.-L.; Hu, H.-Q. A review of models of forest fire occurrence prediction in China. J. Appl. Ecol. 2020, 31, 3227-3240.

12. Ervilha, A.; Pereira, J.; Pereira, J. On the parametric uncertainty quantification of the Rothermel's rate of spread model. Appl. Math. Model. 2016, 41, 37-53. [CrossRef]

13. Zhang, X.-T.; Liu, P.-S.; Wang, X.-F. Research on Improvement of Wang Zhengfei's Forest Fire Spread Model. Shandong For. Sci. Technol. 2020, 50, 1-6.

14. Finney, M. FARSITE: Fire Area Simulator-Model Development and Evaluation; Research Papers RMRS; USDA Forest Service: Washington, DC, USA, 1998.

15. Richards, G. The Properties of Elliptical Wildfire Growth for Time Dependent Fuel and Meteorological Conditions. Combust. Sci. Technol. 1993, 95, 357-383. [CrossRef]

16. Hernandez, E.-L.; White, S.; Rey, Á.; Sánchez, G. Modelling of forest fire spread using Cellular Automata. Appl. Math. Model. 2017, 31, 1213-1227. [CrossRef]

17. Chopard, B. Cellular Automata Modeling of Physical Systems; Springer: New York, NY, USA, 2009.

18. Purnomo, D.; Bonner, M.; Moafi, S.; Rein, G. Using cellular automata to simulate field-scale flaming and smouldering wildfires in tropical peatlands. Proc. Combust. Inst. 2021, 38, 5119-5127. [CrossRef]

19. Darmawan, S.; Sari, D.; Wikantika, K.; Tridawati, A.; Hernawati, R.; Sedu, M. Identification before-after forest fire and prediction of mangrove forest based on Markov-cellular automata in part of Sembilang national park, Banyuasin, South Sumatra, Indonesia. Remote Sens. 2020, 12, 3700. [CrossRef]

20. El, Y.-S.; Jai, A. Cellular automata modelling and spreadability. Math. Comput. Model. 2002, 36, 1059-1074.

21. Trucchia, A.; D’Andrea, M.; Baghino, F.; Fiorucci, P.; Ferraris, L.; Negro, D.; Gollini, A.; Severino, M. PROPAGATOR: An Operational Cellular-Automata Based Wildfire Simulator. Fire 2020, 3, 26. [CrossRef]

22. Mutthulakshmi, K.; Wee, M.-R.-E.; Wong, Y.-C.-K. Simulating forest fire spread and fire-fighting using cellular automata. Chin. J. Phys. 2020, 65, 642-650. [CrossRef]

23. Jellouli, O.; Bernoussi, A.-S.; Maatouk, M.; Amharref, M. Forest fire modelling using cellular automata: Application to the watershed Oued Laou (Morocco). Math. Model. Syst. 2016, 22, 493-507. [CrossRef]

24. Sun, T.; Zhang, L.-H.; Chen, W.-L.; Tang, X.-X.; Qin, Q.-Q. Mountains Forest Fire Spread Simulator Based on Geo-Cellular Automaton Combined With Wang Zhengfei Velocity Model. IEEE J. Sel. Top. Appl. Earth Obs. Remote Sens. 2013, 6, $1971-1987$. [CrossRef]

25. Zhou, G.-X.; Wu, Q.; Chen, A.-B. Forestry Fire Spatial Diffusion Model Based on Multi-Agent Algorithm with Cellular Automata J. Syst. Simul. 2018, 30, 824.

26. Liu, Z.-C.; Zhang, K.; Wang, C.-Y.; Huang, S.-Y. Research on the identification method for the forest fire based on deep learning. Optik- Int. J. Light Electron Opt. 2020, 223, 165491. [CrossRef]

27. Ott, C.; Adhikari, B.; Alexander, S.; Hodza, P.; Chen, X.; Minckley, T. Predicting Fire Propagation across Heterogeneous Landscapes Using WyoFire: A Monte Carlo-Driven Wildfire Model. Fire 2020, 3, 71. [CrossRef]

28. Milanović, S.; Marković, N.; Pamučar, D.; Gigović, L.R; Kostić, P.; Milanović, S.-D. Forest Fire Probability Mapping in Eastern Serbia: Logistic Regression versus Random Forest Method. Forests 2020, 12, 5. [CrossRef]

29. Pimont, F.; Fargeon, H.; Opitz, T. Prediction of regional wildfire activity in the probabilistic Bayesian framework of Firelihood. Ecol. Appl. 2021, 31, e02316. [CrossRef] [PubMed]

30. Zheng, Z.; Huang, W.; Li, S.-N.; Zeng, Y.-N. Forest fire spread simulating model using cellular automaton with extreme learning machine. Ecol. Model. 2017, 348, 33-43. [CrossRef]

31. Hodges, J.L.; Lattimer, B.Y. Wildland Fire Spread Modeling Using Convolutional Neural Networks. Fire Technol. 2019, 55, 2115-2142. [CrossRef]

32. Yang, S.-W.; Lupascu, M.; Meel, K. Predicting Forest Fire Using Remote Sensing Data Furthermore, Machine Learning. arXiv 2021, arXiv:2101.01975.

33. Radke, D.; Hessler, A.; Ellsworth, D.-F. Leveraging Deep Learning to Predict Wildfire Spread. In Proceedings of theTwenty-Eighth International Joint Conference on Artificial Intelligence, Macao, China, 10-16 August 2019.

34. Piyush, J.; Sean, C.-P.; Sriram, G.-S.; Mark, C.; Steve, T.; Mike, D.-F. A review of machine learning applications in wildfire science and management. Environ. Rev. 2020, 28, 478-505. 
35. Lopes, A.; Ribeiro, L.-M.; Viegas, D.-X. Simulation of forest fire spread using a two-way coupling algorithm and its application to a real wildfire. J. Wind. Eng. Ind. Aerodyn. 2019, 193, 103967. [CrossRef]

36. Rong, H.; Pain, C.; Navon, I. Rapid spatio-temporal flood prediction and uncertainty quantification using a deep learning method. J. Hydrol. 2019, 575, 911-920.

37. Fan, S.-T.; Xiao, N.-H.; Dong, S. A novel model to predict significant wave height based on long short-term memory network. Ocean Eng. 2020, 205, 107298. [CrossRef]

38. Sayah, M.; Guebli, D.; Al Masry, Z.; Zerhouni, N. Robustness testing framework for RUL prediction Deep LSTM networks. ISA Trans. 2021, 113, 28-38. [CrossRef]

39. Qing, X.-Y.; Niu, Y.-G. Hourly day-ahead solar irradiance prediction using weather forecasts by LSTM. Energy 2018, 148, 461-468. [CrossRef]

40. Ghodrat, M.; Shakeriaski, F.; Nelson, D.; Simeoni, A. Existing Improvements in Simulation of Fire-Wind Interaction and Its Effects on Structures. Fire 2021, 4, 27. [CrossRef]

41. Liu, J.-G.; Yang, X.-J.; Feng, Y.-Y.; Cui, P. New fractional derivative with sigmoid function as the kernel and its models. Chin. J. Phys. 2020, 68, 533-541. [CrossRef]

42. Liu, J.-K. Chapter 2-Sliding mode control with high performance. In Sliding Mode Control Using MATLAB; Academic Press: Cambridge, MA, USA, 2017; pp. 31-50.

43. Rothermel, B.C. Forest fires and the chemistry of forest fuels. In Thermal Uses and Properties of Carbohydrates and Lignins; Academic Press Inc.: New York, NY, USA, 1976; pp. 245-259.

44. Rui, X.-P.; Hui, S.; Yu, X.-T.; Zhang, G.-Y.; Wu, B. Forest fire spread simulation algorithm based on cellular automata. Nat. Hazards J. Int. Soc. Prev. Mitig. Nat. Hazards 2018, 91, 309-319. [CrossRef]

45. Stijn, V.-D.; Anton, J.-E. Metric distances derived from cosine similarity and Pearson and Spearman correlations. arXiv 2012, arXiv:1208.3145.

46. Luo, C.-J.; Zhan, J.-F.; Wang, L.; Yang, Q. Cosine Normalization: Using Cosine Similarity Instead of Dot Product in Neural Networks. In Proceedings of the International Conference on Artificial Neural Networks, Alghero, Italy, 11-14 September 2017.

47. Hanley, K.; Getmansky, M.; Girardi, G.; Nikolova, S.; Pelizzon, L. Portfolio Similarity and Asset Liquidation in the Insurance Industry. Remote Sens. 2016, 142, 69-96.

48. Bayramov, E.; Buchroithner, M.; Kada, M.N; Zhuniskenov, Y. Quantitative Assessment of Vertical and Horizontal Deformations Derived by 3D and 2D Decompositions of InSAR Line-of-Sight Measurements to Supplement Industry Surveillance Programs in the Tengiz Oilfield (Kazakhstan). Remote Sens. 2021, 13, 2579. [CrossRef]

49. Abo, S.; Oqaibi, H. Using Residual Networks and Cosine Distance-Based K-NN Algorithm to Recognize On-Line Signatures. IEEE Access 2021, 9, 54962-54977.

50. Frandsen, W. Fire spread through porous fuels from the conservation of energy. Combust. Flame 1971, 16, 9-16. [CrossRef]

51. Zhang, J.-Q.; Zhou, M.; Zhao, P.-W.; Shu, Y. Prediction Model of Dead Fuel Load in Burned Area with Different Burning Degree. For. Eng. 2021, 37, 21-27, 35.

52. Schag, G.; Stow, D.; Riggan, P.; Tissell, R.; Coen, J. Examining landscape-scale fuel and terrain controls of wildfire spread rates using repetitive airborne thermal infrared (ATIR) imagery. Fire 2021, 4, 6. [CrossRef]

53. Yu, S.-J.; You, K.; Huang, Q. Visibility Estimation and Forecast in Foggy Weather. Int. Core J. Eng. 2021, 7, 133-145.

54. Yu, J.; Gao, N.; Meng, Z.Z.; Zhang, Z.H. High-accuracy projector calibration method for fringe projection profilometry considering perspective transformation. Opt. Express 2021, 29, 15053-15066. [CrossRef] [PubMed]

55. Kocur, V.; Ftáčnik, M. Detection of 3D bounding boxes of vehicles using perspective transformation for accurate speed measurement. Mach. Vis. Appl. 2020, 31,1-15. [CrossRef]

56. Kim, J. Augmentation of limited input data using an artificial neural network method to improve the accuracy of water quality modeling in a large lake. J. Hydrol. 2021, 602, 142-149. [CrossRef]

57. Shidrokh, G.; Seyed, A.-S.; Mohammad, H.-A.; Domenico, C.; Nazri, K. Real-Time and Intelligent Flood Forecasting Using UAV-Assisted Wireless Sensor Network. CMC—Comput. Mater. Contin. 2022, 70, 715-738.

58. Pham, H.-V.; Trinh, T.-D.; Tieu, K.-M.; Pham, H.-U.; Pham, T.-B. Stock-Price Forecasting Based on XGBoost and LSTM. Comput. Syst. Sci. Eng. 2022, 40, 237-246.

59. Abdulwahed, S.; Abdelaaziz, E. Energy consumption prediction model with deep inception residual network inspiration and LSTM. Math. Comput. Simul. 2021, 190, 97-109.

60. Picotte, J.; Dockter, D.; Long, J.; Tolk, B.; Davidson, A.; Peterson, B. LANDFIRE Remap Prototype Mapping Effort: Developing a New Framework for Mapping Vegetation Classification, Change, and Structure. Fire 2019, 2, 35. [CrossRef]

61. Li, Q.-D.; Xiong, Q.-Y.; Ji, S.-F.; Yu, Y.; Wu, C.; Gao, M. Incremental semi-supervised Extreme Learning Machine for Mixed data stream classification. Expert Syst. Appl. 2021, 185, 115591. [CrossRef]

62. Zeng, G.-P. On the confusion matrix in credit scoring and its analytical properties. Commun. Stat.-Theory Methods 2020, 49 2080-2093. [CrossRef]

63. Simon, D.; Simon, D.-L. Analytic Confusion Matrix Bounds for Fault Detection and Isolation Using a Sum-of-Squared-Residuals Approach. IEEE Trans. Reliab. 2010, 59, 287-296. [CrossRef]

64. Nitish; Amit, K.-S. Automatic detection classification and area calculation of brain tumour in MRI using wavelet transform and SVM classifier. Int. J. Intell. Syst. Technol. Appl. 2021, 19, 526-540. 
65. Gao, M.-Y.; Ni, H.-M.; Zhang, B.-Y. A Method for Recognizing Wood Knots Defects Based on GoogLeNet Convolutional Neural Network. For. Eng. 2021, 37, 66-70.

66. Weng, H.-L.; Wang, S.; Lin, X.-N.; Li, Z.X.; Huang, J.-G. A novel criterion applicable to transformer differential protection based on waveform sinusoidal similarity identification. Int. J. Electr. Power Energy Syst. 2019, 105, 305-314. [CrossRef]

67. Miguel, M.-G.; Germán, B.; Paola, C.-S.; María, L.-T. Increase in the quality of the prediction of a computational wildfire behavior method through the improvement of the internal metaheuristic. Fire Saf. J. 2016, 82, 49-62.

68. Liu, X.-L.; Wu, D.-Y.; Wang, H.-Z.; Liu, J.X. Wetland Type Information Extraction Using Deep Convolutional Neural Network. J. Coast. Res. 2020, 115, 526-529. [CrossRef]

69. Cheng, X.J.; Wan, S.-P.; Dong, J.-Y. A new consistency definition of interval multiplicative preference relation. Fuzzy Sets Syst. 2021, 409, 55-84. [CrossRef]

70. Sahin, B. Consistency control and expert consistency prioritization for FFTA by using extent analysis method of trapezoidal FAHP. Appl. Soft Comput. 2017, 56, 46-54. [CrossRef] 